\title{
Downregulated caveolin-1 expression serves a potential role in coronary artery spasm by inducing nitric oxide production in vitro
}

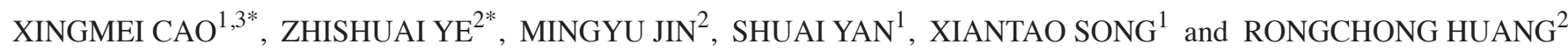 \\ ${ }^{1}$ Department of Cardiology, Beijing An Zhen Hospital, Capital Medical University, Beijing 100029; \\ ${ }^{2}$ Department of Cardiology, The First Affiliated Hospital of Dalian Medical University, Dalian, Liaoning 116011, P.R. China
}

Received May 19, 2017; Accepted August 1, 2017

DOI: $10.3892 /$ etm.2018.6646

\begin{abstract}
The present study aimed to investigate the effects of downregulated caveolin-1 (Cav-1) expression on nitric oxide (NO) production in lipopolysaccharide (LPS)-damaged primary human umbilical vein endothelial cells (HUVECs) in a model of coronary artery spasm (CAS) microenvironment induced by acetylcholine (ACh) treatment. Small interfering RNA (siRNA)-mediated Cav-1 downregulation in HUVECs was confirmed by western blotting. The cell viability and superoxide dismutase (SOD) inhibition in HUVECs incubated with LPS $(0,10,25,50,75$ and $100 \mu \mathrm{g} / \mathrm{ml})$ were measured by cell counting kit- 8 assay and a SOD kit, respectively. Intracellular $\mathrm{Ca}^{2+}\left[\left(\mathrm{Ca}^{2+}\right) \mathrm{i}\right]$ in Fluo4-acetoxymethyl ester-loaded cells was detected by fluorescence microscopy. NO levels in the cell culture supernatants were measured by the nitrate reductase method. The results indicated that transfection with Cav-1 siRNA, in particular siCav-1 (2), downregulated the Cav-1 protein expression. LPS at a dose of $75 \mu \mathrm{g} / \mathrm{ml}$ induced a significant decrease in HUVECs/si-NC and HUVECs/siCav-1 viability compared with the other concentrations of LPS. Compared with the effects of untreated cells, SOD inhibition in HUVECs/si-NC and HUVECs/siCav-1 was significantly decreased by LPS $(75 \mu \mathrm{g} / \mathrm{ml})$. In addition, ACh stimulation caused a greater increase in $\left[\mathrm{Ca}^{2+}\right] \mathrm{i}$ in $\mathrm{HUVECs} / \mathrm{si}-\mathrm{NC}$ as
\end{abstract}

Correspondence to: Professor Xiantao Song, Department of Cardiology, Beijing An Zhen Hospital, Capital Medical University, 2 Anzhen Road, Chaoyang, Beijing 100029, P.R. China

E-mail: songxiantao@medmail.com.cn

Professor Rongchong Huang, Department of Cardiology, The First Affiliated Hospital of Dalian Medical University, 222 Zhongshan Road, Xigang, Dalian, Liaoning 116011, P.R. China

E-mail: rchuang@dlmedu.edu.cn

Present address: ${ }^{3}$ Department of Cardiology, Daxing Hospital, Capital Medical University, Beijing 102600, P.R. China

*Contributed equally

Key words: caveolin-1, nitric oxide, lipopolysaccharide, acetylcholine, coronary artery spasm compared with LPS-treated HUVECs/si-NC. ACh stimulation also induced significantly higher NO levels in LPS-treated HUVECs/siCav-1 compared with LPS-treated HUVECs/si-NC cells $(\mathrm{P}<0.05)$.Inconclusion, the downregulated Cav-1 expression served a key role in NO production in the in vitro model of CAS induced by ACh stimulation of LPS-damaged HUVECs.

\section{Introduction}

Coronary artery spasm (CAS) serves an important role in the pathogenesis of numerous myocardial ischemic disease, including stable angina, unstable angina, acute myocardial infarction and sudden cardiac death $(1,2)$. The pathogenesis of CAS occurs through a complex mechanism that involves endothelial dysfunction, inflammation, hyperactivity of smooth muscle cells and other factors $(3,4)$. However, the specific regulation of CAS remains to be elucidated.

Nitric oxide (NO) is generated from L-arginine by constitutive endothelial NO synthase (eNOS) (5). In the vasculature, the bioactivity of eNOS and NO is regulated by the caveolins, which are scaffolding/regulatory proteins that are particularly abundant in the endothelial cell plasma membrane $(6,7)$. Caveolin-1 (Cav-1) is the most important caveolar coat protein involved in the control of vascular reactivity by combining with eNOS (8). eNOS binds to Cav-1 via the caveolin scaffolding domain, which is located at amino acids 350-358 (9). This interaction leads to the inhibition of eNOS activity, resulting in a decrease in the basal release of NO (10) and increase in the cardiovascular tone (11).

Endothelial dysfunction is characterized mainly by a reduction in eNOS-derived NO production and bioactivity (12), as well as an imbalance between the endothelium-derived relaxation factor and vasoconstrictors. Previous studies have demonstrated endothelial dysfunction at the site of a CAS (13). Therefore, maintenance of the release and bioactivity of NO in the endothelial environment is important in preventing CAS. Previous studies have demonstrated that eNOS becomes hyperactivated in the absence of Cav-1, leading to marked vascular relaxation $(12,14,15)$. Thus, CAS may be caused by decreased NO release as a result of eNOS inhibition by Cav-1 in certain pathological conditions. This negative regulation is particularly important since eNOS activation has been associated with a protective effect against the development of CAS. However, the potential of Cav-1 knockdown to alleviate or 
inhibit the development of CAS remains to be investigated. It can be speculated that Cav-1 knockdown in endothelial cells may produce high levels of bioactive $\mathrm{NO}$, which regulates the vascular tone in order to inhibit the occurrence of CAS.

In the current study, an in vitro model of endothelial damage stimulated by lipopolysaccharide (LPS) treatment was established, and then the CAS environment was mimicked by the addition of acetylcholine (ACh) to investigate the effects of Cav-1 knockdown. It was hypothesized that Cav-1 knockdown in this LPS-induced model of endothelial cell dysfunction may increase ACh-stimulated NO production. It was observed that the high levels of NO induced by Cav-1 knockdown serve a vital role in the inhibition of CAS development. Therefore, targeting the interaction between CAS and Cav-1 represents a potential therapeutic strategy for CAS.

\section{Materials and methods}

Culture and identification of HUVECs. Primary human umbilical vein endothelial cells (HUVECs; ScienCell Research Laboratories, Inc., Carlsbad, CA, USA) were maintained in endothelial cell medium (ECM; ScienCell Research Laboratories, Inc.) containing 5\% fetal bovine serum (FBS), $1 \%$ endothelial cell growth supplement and $1 \%$ penicillin/streptomycin. The cells were incubated at $37^{\circ} \mathrm{C}$ in a humidified atmosphere with $5 \% \mathrm{CO}_{2}$. In all experiments, cells at $90 \%$ confluence and between passages 3 and 5 were used. The cells were characterized as endothelial cells according to their morphology and factor VIII staining using an anti-factor VIII antibody (cat. no. 21458-1-AP; ProteinTech Group, Inc., Wuhan, China), which is a well-recognized marker of endothelial cells. The medium was aspirated; the cells were washed with PBS seeded on clean glass coverslips. The cells were fix with freshly made $4 \%$ paraformaldehyde (Servicebio, Wuhan, China) in PBS at room temperature for $1 \mathrm{~h}$. The coverslips were rinsed with PBS three times (5 min/wash) and treated with $0.1 \%$ TritonX-100 (cat. no. T8200; Beijing Solarbio Science \& Technology Co., Ltd., Beijing, China) in PBS for $15 \mathrm{~min}$ at room temperature. The coverslips were rinsed with PBS three times (5 min/wash). The cells were then blocked in $10 \%$ normal blocking goat serum in PBS at room temperature for $30 \mathrm{~min}$. The blocking solution was aspirated and the cells were incubated with the rabbit anti-factor VIII antibodies $(1: 100)$ for $4 \mathrm{~h}$ at $4^{\circ} \mathrm{C}$. The coverslips were rinsed with PBS four times (5 min/wash). Then the cells were incubated with fluorescein isothiocyanate-conjugated goat anti-rabbit immunoglobulin G (1:100; cat. no. ZF-0311; ZSGB-Bio; OriGene Technologies, Inc., Beijing, China) for $30 \mathrm{~min}$ at room temperature in a dark, moist environment. The coverslips were rinsed with PBS three times ( $5 \mathrm{~min} /$ wash). The coverslip were then incubated with DAPI (cat. no. C1005; Beyotime Institute of Biotechnology, Jiangsu, China) to stain the nuclei and cells were examined using a fluorescence microscope (magnification, x200).

Experimental groups. Preliminary experiments demonstrated that significant cellular damage is caused by pretreatment of HUVECs with $75 \mu \mathrm{g} / \mathrm{ml}$ LPS (serotype 026:B6 from Escherichia coli; Sigma-Aldrich; Merck KGaA, Darmstadt, Germany) for $24 \mathrm{~h}$ at $37^{\circ} \mathrm{C}$. Subsequently, these cells were treated with $10 \mu \mathrm{M}$ ACh (Sigma-Aldrich; Merck KGaA) for $1 \mathrm{~h}$ at $37^{\circ} \mathrm{C}$. The cells were then divided into the following experimental groups: i) HUVECs/si-NC, which was a negative control for Cav-1 downregulation; ii) HUVECs/si-NC + LPS; iii) HUVECs/si-NC + ACh; iv) HUVECs/si-NC + LPS + ACh; v) HUVECs/siCav-1, in which Cav-1 downregulation was performed; vi) $\mathrm{HUVECs} / \mathrm{siCav}-1$ + LPS; vii) $\mathrm{HUVECs} / \mathrm{siCav}-1$ + ACh; and viii) HUVECs/siCav-1 + LPS + ACh. Subsequently, following all the treatments, the cell culture supernatants were centrifuged at $1,800 \mathrm{x} \mathrm{g}$ for $10 \mathrm{~min}$ at $4^{\circ} \mathrm{C}$ to remove any cell debris.

Transfection and Lipofectamine assay. Small interfering RNA (siRNA) duplex oligonucleotides that were specific for Cav-1 were synthesized by Suzhou GenePharma Co., Ltd. (Suzhou; China). The sequences were as follows: siCav-1 (1), 5'-GCA UUUGGAAGGCCAGCUUTT-3'; siCav-1 (2), 5'-CCCACU CUUUGAAGCUGUUTT-3'; siCav-1 (3), 5'-GCAGUUGUA CCAUGCAUUATT-3'; and si-NC, 5'-UUCUCCGAACGU GUCACGUTT-3'. For transfection, cells $\left(2 \times 10^{5}\right.$ cells/well $)$ were seeded into 6-well culture plates at 50-60\% confluence. After $24 \mathrm{~h}$, siRNA (30 pmol) mixed with $5 \mu$ l Lipofectamine RNAiMAX reagent (Invitrogen; Thermo Fisher Scientific, Inc., Waltham, MA, USA) in $250 \mu 1$ serum-free Opti-MEM (Gibco; Thermo Fisher Scientific, Inc.) was added to each well and incubated for $15 \mathrm{~min}$ at room temperature, followed by addition of $2.5 \mathrm{ml}$ ECM without serum. After $4-6 \mathrm{~h}$, the transfection solution was replaced with the complete culture medium and incubated for a further $48 \mathrm{~h}$ at $37^{\circ} \mathrm{C}$. Subsequently, the cells were harvested and the knockdown efficiency was evaluated by western blotting.

Western blot analysis of siRNA transfection efficiency. Cells were lysed in radioimmunoprecipation assay (RIPA) buffer and phenylmethylsulfonyl fluoride (Beyotime Institute of Biotechnology, Jiangsu, China) for $15 \mathrm{~min}$ on ice. The total cell lysates were centrifuged at $12,000 \mathrm{xg}$ for $15 \mathrm{~min}$ at $4^{\circ} \mathrm{C}$, and the protein concentration was measured using the BCA Protein Assay reagent (Beyotime Institute of Biotechnology). Proteins were denatured by adding $1 \mathrm{X}$ sodium dodecyl sulfate (SDS; Beyotime Institute of Biotechnology) loading buffer and boiled for $10 \mathrm{~min}$. Next, the protein samples $(\sim 10 \mu \mathrm{g})$ were separated by $12 \%$ SDS-polyacrylamide gel electrophoresis and transferred to nitrocellulose membranes (Pall Corporation, Dreieich, Germany). The blots were then blocked for $2 \mathrm{~h}$ at room temperature with $5 \%$ non-fat dried milk, and subsequently incubated overnight at $4{ }^{\circ} \mathrm{C}$ with the following primary detection antibodies: Rabbit polyclonal anti-CAV-1 (1:1,500; cat. no. sc-894; Santa Cruz Biotechnology Inc., Dallas, TX, USA) and rabbit polyclonal anti-GAPDH antibody (1:2,000; cat. no. BS60630; Bioworld Technology, Co, Ltd., Nanjing, China). Subsequent to washing three times (15 min per wash) with Tris-buffered saline and $0.05 \%$ Tween-20, the blots were incubated with a goat anti-rabbit secondary detection antibody (1:4,000; cat. no. ZDR-530612; ZSGB-Bio; OriGene Technologies, Inc.) for $60 \mathrm{~min}$ at room temperature. Following further washing, the immunoreactivity was visualized with an enhanced chemiluminescence kit (Advansta, Inc., Menlo Park, CA, USA). Relative quantification of proteins was performed using ImageLab software (version 4.0; Bio-Rad 
Laboratories, Inc., Hercules, CA, USA). Three independent experiments were performed for each analysis.

Cell counting kit-8 (CCK-8) assay of LPS-treated HUVEC viability. In order to induce endothelial cell injury, HUVECs were incubated with bacterial LPS, and the cell viability was assessed using the CCK-8 assay (Dojindo Molecular Technologies, Inc., Kumamoto, Japan). The HUVECs/si-NC and HUVECs/siCav-1 were plated in flat-bottom 96-well plates $\left(5 \times 10^{3}\right.$ cells/well) and allowed to adhere for $24 \mathrm{~h}$ at $37^{\circ} \mathrm{C}$. Cells were then treated with various concentrations of LPS $(0,10$, $25,50,75$ and $100 \mu \mathrm{g} / \mathrm{ml}$ ) for $24 \mathrm{~h}$ at $37^{\circ} \mathrm{C}$ in ECM containing $2.5 \%$ FBS, while PBS was added to cells in the control group. After $24 \mathrm{~h}$, the medium was removed, and the CCK-8 solution was added $(10 \mu 1 \mathrm{CCK}-8+90 \mu \mathrm{l}$ ECM) to each well. The plates were then incubated for $2 \mathrm{~h}$ and the absorbance was detected at $450 \mathrm{~nm}$ in a microplate reader.

Measurement of superoxide dismutase (SOD) inhibition. SOD inhibition was measured using the SOD Assay kit-WST kit (Dojindo Molecular Technologies, Inc.) according to manufacturer's protocol. Briefly, HUVECs were seeded in 6 -well plates $\left(1 \times 10^{5}\right.$ cells/well) for $24 \mathrm{~h}$ prior to the addition of LPS $(75 \mu \mathrm{g} / \mathrm{ml})$. After $24 \mathrm{~h}$, the cells were lysed in RIPA buffer and centrifuged at $10,000 \mathrm{x}$ g for $15 \mathrm{~min}$ at $4^{\circ} \mathrm{C}$. The supernatants, enzyme-working solution and WST solution were added to 96-well plates according to the manufacturer's protocol. Finally, the absorbance was measured at $450 \mathrm{~nm}$ with a microplate reader.

Measurement of a fluorescent molecular probe for analysis of intracellular $\mathrm{Ca}^{2+}\left[\left(\mathrm{Ca}^{2+}\right) i\right]$. To monitor alterations in the $\left[\mathrm{Ca}^{2+}\right] \mathrm{i}$ levels in response to ACh treatment $(10 \mu \mathrm{M})$, cells were loaded with the fluorescent dye Fluo4-acetoxymethyl ester (Fura4-AM; Dojindo Molecular Technologies, Inc.). Briefly, cells were seeded into a culture chamber for $24 \mathrm{~h}$ at $37^{\circ} \mathrm{C}$, and LPS was added for a further $24 \mathrm{~h}$, followed by incubation with Fura4-AM $(5 \mu \mathrm{M})$ for $30 \mathrm{~min}$ at $37^{\circ} \mathrm{C}$. Subsequent to removing the medium, cells were covered with Hank's balanced salt solution with $\mathrm{Ca}^{2+}$ (cat. no. CC014; Macgene, Biotechnology Ltd., Beijing, China), and visualized under a fluorescence microscope (IX 70; Olympus Corp., Hamburg, Germany) at an excitation wavelength of $488 \mathrm{~nm}$ and emission wavelength of $512 \mathrm{~nm}$. Images were acquired at $1 \mathrm{sec}$ intervals for $300 \mathrm{sec}$. Alterations in the fluorescence reflecting changes in $\left[\mathrm{Ca}^{2+}\right] \mathrm{i}$ were presented as the integrated optical density and were quantified using Image-Pro Plus version 6.0 software (Media Cybernetics, Inc., Rockville, MD, USA).

Nitrate reductase method for the detection of the NO content in cells. The NO content in HUVECs/si-NC and HUVECs/siCav-1 treated with or without LPS and/or Ach was also investigated. The culture supernatants were collected from all eight experimental groups following treatment. Next, NO concentrations were measured using a nitrate reductase method with an NO detection kit (Nanjing Jiancheng Bioengineering Institute, Nanjing, China) according to the manufacturer's protocol.

Statistical analysis. All data are presented as the mean \pm standard deviation. Relative Cav-1/GAPDH protein expression levels, HUVEC viability and SOD inhibition were analyzed by one-way analysis of variance for multiple-group comparisons. When a statistical difference was observed, the data were further analyzed using Bonferroni's post-hoc test with GraphPad Prism version 5 software (GraphPad Software, Inc., La Jolla, CA, USA). P $\leq 0.05$ was considered to indicate differences that were statistical significance.

\section{Results}

Characterization of cultured HUVECs. The primary HUVECs started to attach to the plates subsequent to culture for $\sim 1 \mathrm{~h}$, demonstrating a typical cobblestone-like appearance under the inverted microscope and positive immunofluorescent staining with anti-factor VIII antibody (Fig. 1). Thus, these cells were confirmed to be HUVECs.

siRNA-mediated downregulation of Cav-1 expression in HUVECs. Western blot analysis revealed that, among the three double-stranded siRNAs investigated, siCav-1(2) transfection presented the best efficiency at inducing a reduction in Cav-1 protein expression compared with the with HUVECs group ( $70 \%$ reduction; $\mathrm{P}<0.001$; Fig. 2). Thus, siCav-1(2) was used in all subsequent experiments in the present study.

LPS-induced a reduction in HUVEC viability. Following the incubation of HUVECs/si-NC and HUVECs/siCav-1 with different concentrations of LPS for $24 \mathrm{~h}$, CCK 8 analysis demonstrated that the LPS-induced cell viability was decreased in a dose-dependent manner. There were no significant alterations in the viability of HUVECs/si-NC and HUVECs/siCav-1 following treatment with LPS at the lower concentrations (10, 25 and $50 \mu \mathrm{g} / \mathrm{ml}$ ). However, compared with the untreated cells, a significant decrease in cell viability was observed following treatment of HUVECs/si-NC and HUVECs/siCav-1 with LPS at 75 and $100 \mu \mathrm{g} / \mathrm{ml}(\mathrm{P}<0.001)$. Treatment with LPS at 75 and $100 \mu \mathrm{g} / \mathrm{ml}$ for $24 \mathrm{~h}$ resulted in respective cell viabilities of 69.06 and $15.56 \%$ in the HUVECs/si-NC, and 72.90 and $13.02 \%$ in the HUVECs/siCav-1. When $100 \mu \mathrm{g} / \mathrm{ml}$ was used, the cell viabilities were so the subsequent experiments would have been detrimentally affected, therefore this concentration was not selected. There was no significant difference in the viability of the HUVECs/si-NC and HUVECs/siCav-1 groups when treated with LPS at $75 \mu \mathrm{g} / \mathrm{ml}$; thus, $75 \mu \mathrm{g} / \mathrm{ml}$ LPS was used in all subsequent experiments (Fig. 3).

LPS promoted HUVEC damage through SOD inhibition. SOD inhibition, which reflects cell damage, was determined in order to evaluate the effects of LPS on HUVECs (Fig. 4). SOD inhibition of 55.45 and $75.36 \%$ was observed in HUVECs/si-NC following treatment with and without LPS, respectively. Similarly, SOD inhibition of 48.28 and $75.05 \%$ was observed in HUVECs/siCav-1 following treatment with and without LPS, respectively. Thus, SOD inhibition was significantly decreased in the presence of LPS $(\mathrm{P}<0.05)$, indicating that LPS $(75 \mu \mathrm{g} / \mathrm{ml})$ caused injury in HUVECs/siCav-1 and HUVECs/siCav-1.

$\left[\mathrm{Ca}^{2+}\right]$ i levels in response to $\mathrm{ACh}$. Alterations in $\left[\mathrm{Ca}^{2+}\right] \mathrm{i}$ were measured in HUVECs/si-NC with or without LPS treatment prior to and following the addition of Ach, used as a 


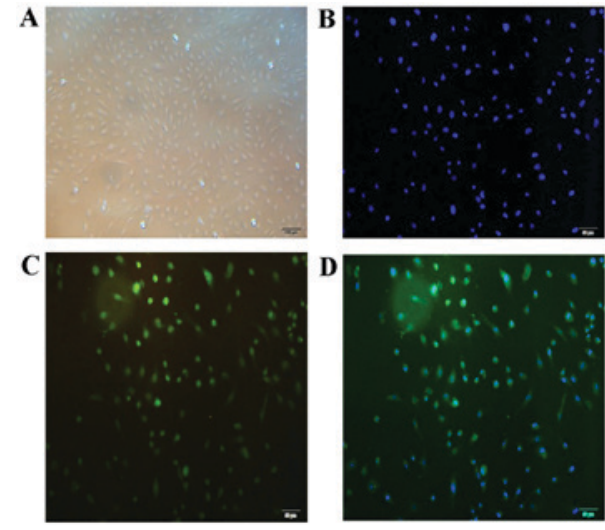

Figure 1. Morphological appearance and identification of HUVECs under the inverted microscope. (A) Cells demonstrated a typical 'cobblestone' pattern without staining (magnification, x100). (B) Nuclei were stained with DAPI (magnification, x200). (C) Immunofluorescent staining of factor VIII expressed in cells (magnification, x200). (D) Merged image of DAPI and factor VIII staining (magnification, x200). HUVECs, human umbilical vein endothelial cells; DAPI, 4',6-diamidino-2-phenylindole.
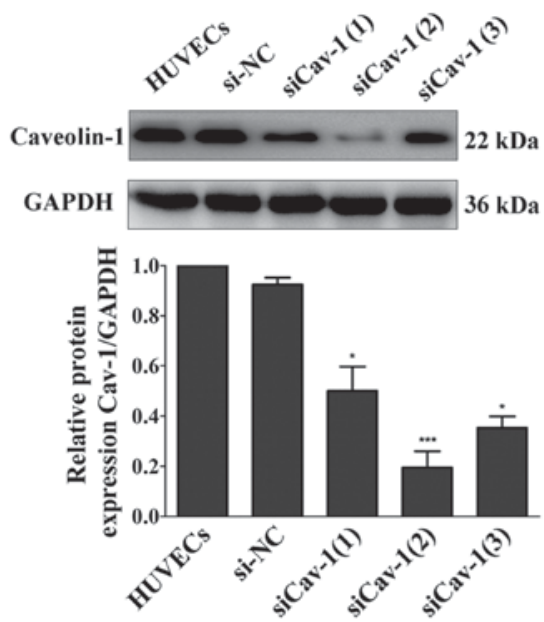

Figure 2. Western blot analysis of Cav-1 protein expression in HUVECs transfected with siCav-1(1), siCav-1(2) and siCav-1(3). GAPDH was used as a loading control. ImageLab software was used to quantify the immunoreactive band density, and GraphPad Prism version 5 software was used to generate the histogram. ${ }^{*} \mathrm{P}<0.05$ and ${ }^{* * *} \mathrm{P}<0.001$ vs. HUVEC. HUVECs, human umbilical vein endothelial cells; Cav-1, caveolin-1; GAPDH, glyceraldehyde 3-phosphate dehydrogenase; si, small interfering RNA; NC, negative control.

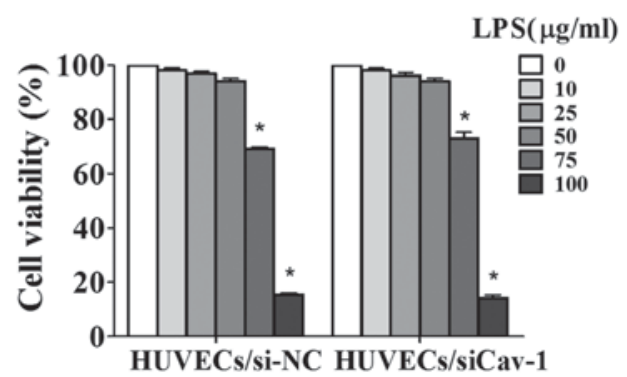

Figure 3. Effects of treatment with LPS $(10-100 \mu \mathrm{g} / \mathrm{ml})$ for $24 \mathrm{~h}$ on the viability of HUVECs/si-NC and HUVECs/siCav-1. Treatment with 75 and $100 \mu \mathrm{g} / \mathrm{ml}$ LPS induced a significant decrease in the viability of HUVECs/si-NC and HUVECs/siCav-1. There was no significant difference in the viability of HUVECs/si-NC and HUVECs/siCav-1 treated with LPS at 75 or $100 \mu \mathrm{g} / \mathrm{ml}$. ${ }^{*} \mathrm{P} \leq 0.001$ vs. absence of LPS treatment. HUVECs, human umbilical vein endothelial cells; LPS, lipopolysaccharide; Cav-1, caveolin-1; si, small interfering RNA; NC, negative control.

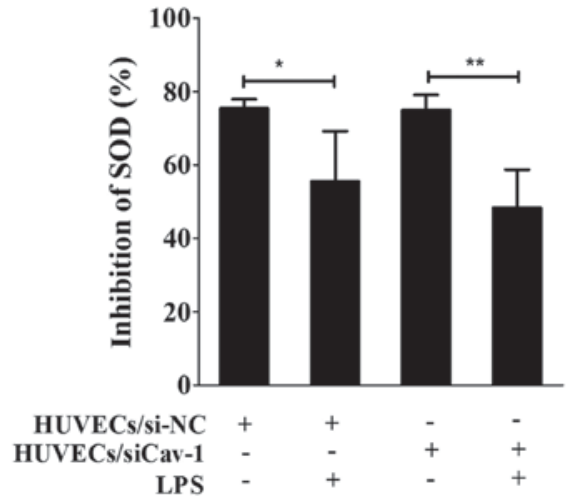

Figure 4. Effect of LPS $(75 \mu \mathrm{g} / \mathrm{ml})$ treatment on SOD activity in HUVECs. LPS treatment significantly decreased the inhibition of SOD in HUVECs/si-NC and HUVECs/siCav-1. ${ }^{*} \mathrm{P} \leq 0.05$ and ${ }^{* *} \mathrm{P} \leq 0.01$. LPS, lipopolysaccharide; SOD, superoxide dismutase; HUVECs, human umbilical vein endothelial cells; Cav-1, caveolin-1; si, small interfering RNA; NC, negative control.

marker of the CAS environment mimicked in vitro. In the HUVECs/si-NC group without LPS treatment, a rapid increase in $\left[\mathrm{Ca}^{2+}\right] \mathrm{i}$ was induced, reaching a maximum followed by a sustained plateau after the addition of Ach (Fig. 5A). Certain cells also demonstrated a $\left[\mathrm{Ca}^{2+}\right] \mathrm{i}$ oscillation phenomenon (16). In the HUVECs/si-NC group treated with LPS, a smaller peak and plateau of $\left[\mathrm{Ca}^{2+}\right]$ i was observed, with certain cells presenting no response to treatment with Ach (Fig. 5B). Thus, the $\left[\mathrm{Ca}^{2+}\right] \mathrm{i}$ was increased in the untreated HUVECs/si-NC group, but was unaltered in the LPS-treated cells, indicating that the LPS-induced injury influenced the level of $\left[\mathrm{Ca}^{2+}\right] \mathrm{i}$ following ACh stimulation and that a CAS environment was successfully mimicked in vitro.

Downregulated Cav-1 expression enhances NO production in LPS-treated HUVECs stimulated with Ach. A significant difference in NO content was observed between the ACh-stimulated HUVECs/si-NC groups with and without LPS treatment $(\mathrm{P}<0.01)$. In the group without LPS treatment, the expression of NO was significantly higher in comparison with that in the LPS-treated group $(4.161 \pm 1.224$ vs. $1.679 \pm 1.049 \mu \mathrm{mol} / 1$, respectively; $\mathrm{P}<0.05$; Fig. 6). Similarly, in the HUVECs/siCav-1 group, the content of NO in the ACh-stimulated group without LPS treatment was significantly higher compared with that in the group treated with LPS $(\mathrm{P}<0.001)$. In the absence of LPS pretreatment, there was a significant difference in the NO expression between the HUVECs/si-NC and HUVECs/siCav-1 groups following ACh stimulation $(\mathrm{P}<0.001)$. Following ACh stimulation, the NO content in LPS-treated HUVECs/siCav-1 was significantly higher when compared with that in the LPS-induced HUVECs/si-NC cells (3.869 \pm 0.7679 vs. $1.241 \pm 0.3674 \mu \mathrm{mol} / \mathrm{l}$, respectively; $\mathrm{P}<0.05$; Fig. 6). No significant difference in $\mathrm{NO}$ content was identified in LPS-treated HUVECs/si-NC with or without ACh. Additionally, no significant difference in NO content was identified in LPS-treated HUVECs/siCav-1 with or without ACh.

\section{Discussion}

In the current study, a CAS microenvironment model for endothelial cells was successfully established as observed 

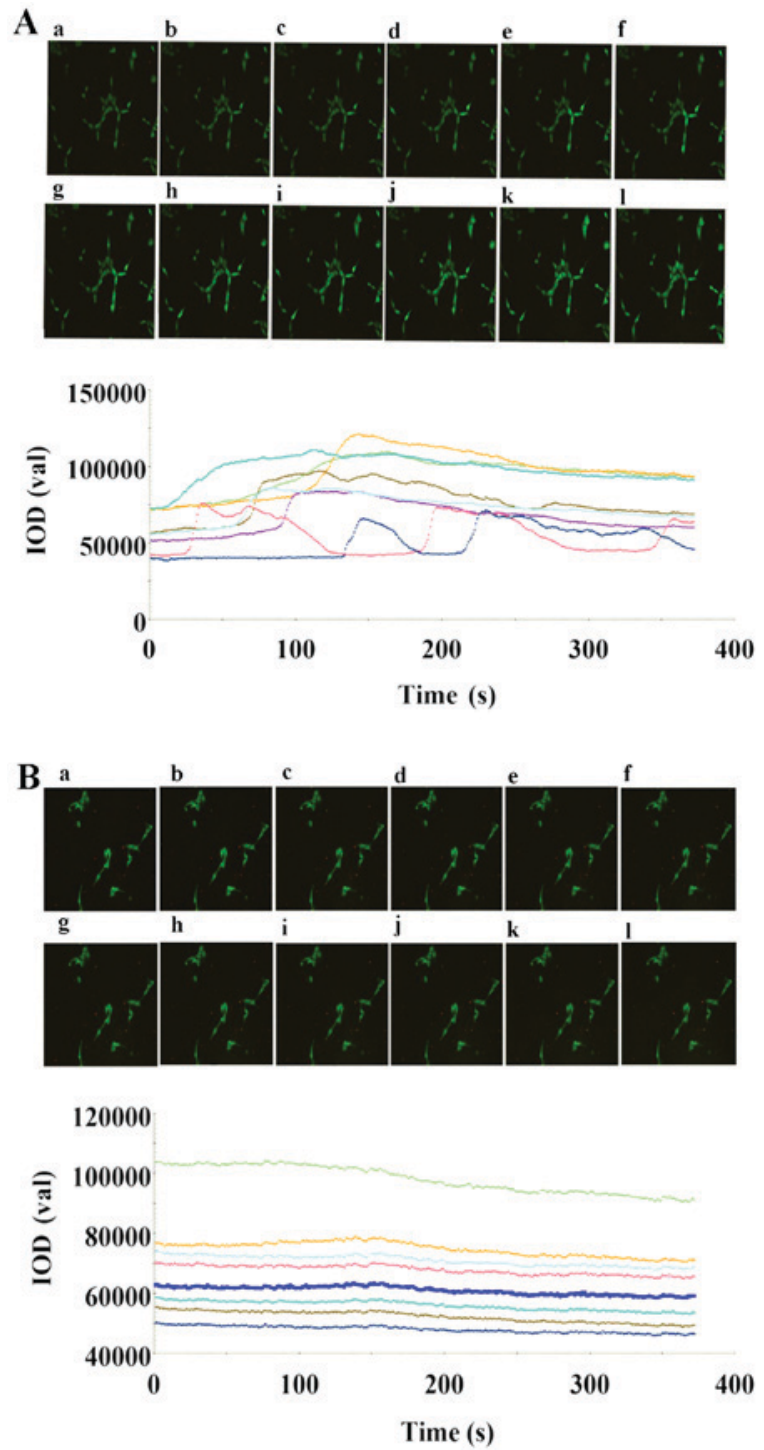

Figure 5. Effects of $10 \mu \mathrm{M}$ Ach added at $10 \mathrm{sec}$ on the $\left[\mathrm{Ca}^{2+}\right] \mathrm{i}$ responses in Fluo4-acetoxymethyl ester-loaded HUVECs/si-NC with or without LPS treatment. The responses in the eight cell groups were evaluated. The ACh-stimulated changes in the $\left[\mathrm{Ca}^{2+}\right]$ i fluorescence of $\mathrm{HUVECs} / \mathrm{si}-\mathrm{NC}$ are shown in the (A) absence and (B) presence of LPS, at the following time points: (a) $1 \mathrm{sec}$; (b) $17 \mathrm{sec}$; (c) $35 \mathrm{sec}$; (d) $70 \mathrm{sec}$; (e) $99 \mathrm{sec}$; (f) $122 \mathrm{sec}$; (g) $158 \mathrm{sec}$; (h) $199 \mathrm{sec}$; (i) $212 \mathrm{sec}$; (j) $232 \mathrm{sec}$; (k) $268 \mathrm{sec}$; and (l) $334 \mathrm{sec}$ In HUVECs/si-NC without LPS treatment, a characteristic biphasic $\left[\mathrm{Ca}^{2+}\right]$ response was observed following the addition of $\mathrm{ACh}(10 \mu \mathrm{M})$ at $10 \mathrm{sec}$, with an initial rapid increase in $\left[\mathrm{Ca}^{2+}\right] \mathrm{i}$, reaching a maximum level at a different time-points for every cell, followed by a sustained plateau in $\left[\mathrm{Ca}^{2+}\right]$ i that declined slowly toward the baseline. In addition, a $\left[\mathrm{Ca}^{2+}\right]$ i oscillation phenomenon was observed with peak and plateau levels occurring regularly. In the HUVECs/si-NC group with LPS treatment, the addition of ACh $(10 \mu \mathrm{M})$ at $10 \mathrm{sec}$ resulted in a smaller peak and plateau compared with those observed in the group without LPS treatment, with certain cells not presenting any response. $\left[\mathrm{Ca}^{2+}\right] \mathrm{i}$, intracellular $\mathrm{Ca}^{2+}$; LPS, lipopolysaccharide; ACh, acetylcholine; HUVECs, human umbilical vein endothelial cells; Cav-1, caveolin-1; si, small interfering RNA; NC, negative control.

by the alterations in $\left[\mathrm{Ca}^{2+}\right] \mathrm{i}$, which was used as an indicator. ACh stimulation increased the $\left[\mathrm{Ca}^{2+}\right]$ i levels in cells that were not damaged by LPS pretreatment, while there was little or no change in $\left[\mathrm{Ca}^{2+}\right]$ i following ACh exposure in LPS-treated HUVECs. These results demonstrated that Cav-1 served an important regulatory role in this process and that downregulation of Cav-1 promotes the release of NO in LPS-treated

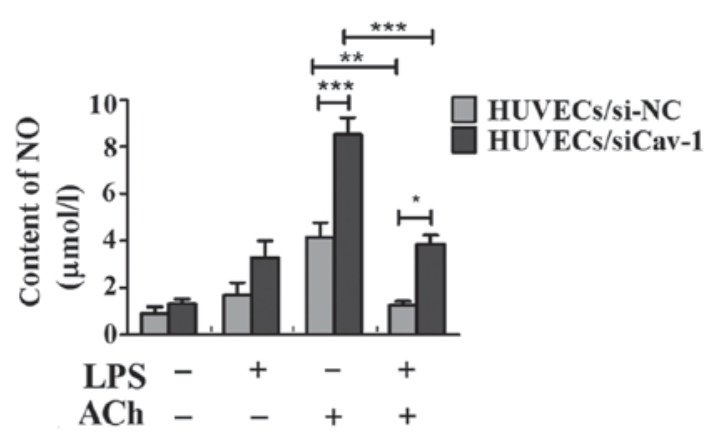

Figure 6. Production of NO with or without ACh exposure in HUVECs/si-NC and HUVECs/siCav-1 with or without LPS pretreatment. ACh stimulated the production of NO in HUVECs/si-NC and HUVECs/siCav-1. The NO level was lower when cells were treated with LPS compared with the levels observed without LPS treatment. ACh stimulated the LPS-induced HUVECs/siCav-1 to produce significantly higher NO levels compared with those produced by LPS-induced HUVECs/si-NC. ${ }^{*} \mathrm{P} \leq 0.05,{ }^{* *} \mathrm{P} \leq 0.01,{ }^{* * *} \mathrm{P} \leq 0.001$. NO, nitric oxide; HUVECs, human umbilical vein endothelial cells; LPS, lipopolysaccharide; ACh, acetylcholine; Cav-1, caveolin-1; si, small interfering RNA; $\mathrm{NC}$, negative control; IOD, integrated optical density.

HUVECs stimulated with ACh. In the CAS microenvironment the production of NO was increased by knocking down Cav-1.

The presence of an intact endothelium is essential for ACh to induce dilation of isolated arteries by NO release. It has been observed that ACh stimulation induces arterial constriction in the absence of the endothelium, due to a reduced bioavailability of NO resulting from its decreased formation or accelerated degradation $(5,17)$. The current study demonstrated that when the endothelium was damaged, ACh treatment did not increase the $\left[\mathrm{Ca}^{2+}\right] \mathrm{i}$; however, the inhibitory role of the Cav-1/eNOS complex cannot be dismissed. The endothelium-dependent agonists, ACh and bradykinin, increase $\left[\mathrm{Ca}^{2+}\right] \mathrm{i}$ and then increase the $\mathrm{Ca}^{2+} /$ calmodulin complex, which in turn, activates eNOS (18). The elevation of $\left[\mathrm{Ca}^{2+}\right] \mathrm{i}$ in endothelial cells induced by $\mathrm{ACh}$ is the result of $\mathrm{Ca}^{2+}$ release from intracellular stores and transmembrane $\mathrm{Ca}^{2+}$ influx $(19,20)$. The endothelial target for ACh is widely expressed in the vascular endothelium, and the endothelium-dependent relaxation mediated by the receptor is considered to be the classical indicator of the endothelial dysfunction $(21,22)$. It has been demonstrated that the expression and bioactivity of the endothelial target for ACh is decreased by endothelial damage. In addition, Cav-1/eNOS binding can be reversed by calcium influx and increased $\mathrm{Ca}^{2+} /$ calmodulin complex formation (23). Therefore, ACh can initiate endothelial cell signaling transduction, leading to decreased NO formation via numerous pathways, including the $\mathrm{Ca}^{2+} /$ calmodulin signaling pathway (24). In vitro models of spasm in arteries exposed to ACh and pharmacological provocation tests using $\mathrm{ACh}$ or ergonovine are well established and have been widely used in the diagnosis of coronary spastic angina (25). However, there are few reports that describe the use of endothelial cells in a model of the CAS microenvironment.

Numerous studies have generated models of coronary spasm using isolated arteries, which contain endothelial and smooth cells; thus, these models are not suitable for investigating the roles of the different cell types. Therefore, for improved investigation of the role of Cav-1 in endothelial cells, it is hypothesized that the alterations in $\left[\mathrm{Ca}^{2+}\right] \mathrm{i}$ in LPS-damaged cells stimulated with ACh can be considered 
as a marker the reflects the CAS microenvironment in vitro. In the present study, ACh stimulation induced a less marked increase in $\left[\mathrm{Ca}^{2+}\right] \mathrm{i}$ in LPS-damaged HUVECs when compared with that induced in cells without LPS treatment. This observation supports the hypothesis that the changes in $\left[\mathrm{Ca}^{2+}\right] \mathrm{i}$ are a suitable marker for the status of the CAS microenvironment.

In the present study, it was observed that the siRNA-mediated downregulation of Cav-1 increased the production of NO when LPS-damaged HUVECs were exposed to ACh. This highlighted Cav-1 inhibition as a potential therapeutic target that may promote NO levels in CAS. These effects are consistent with a previous study that revealed significantly higher basal NO release in Cav-1 knockout mice when compared with that in wild-type mice (26). Furthermore, in the absence of Cav-1, arteries exhibited a lack of steady contractile tone as well as increased relaxation with high NO generation following ACh stimulation (26).

The current study demonstrated that Cav-1 downregulation increased the release of NO when LPS-damaged HUVECs were stimulated by ACh. However, the limitations of the study should be noted. The molecular mechanism of signal transduction is complicated and the marker used in the model employed in the present study requires further investigation. Although the results of the study indicated the regulatory influence of Cav-1 on NO, a more mechanistic understanding is necessary to fully clarify how Cav-1 influences eNOS and NO following ACh stimulation. Furthermore, the present study was based on an in vitro system and since Cav-1 serves a vital role in cells, the degree of Cav-1 knockdown requires further investigation to prevent the occurrence of CAS without affecting other Cav-1 functions.

In conclusion, the present study revealed that LPS and ACh stimulation downregulated Cav-1 expression in a CAS microenvironment, which may serve a key role in NO production. Therefore, Cav-1 may be a potential therapeutic target in an efficient management of coronary spasm.

\section{Acknowledgements}

The abstract of the current study was accepted by the 22nd World conference of 2017 International Academy of Cardiology (Vancouver, BC, Canada) as an oral presentation.

\section{Funding}

The current study was funded by the National Natural Science Foundation of China (grant no. 81670324).

\section{Availability of data and materials}

The datasets used and/or analyzed during the study are available from the corresponding authors on reasonable request.

\section{Authors' contributions}

$\mathrm{XC}$ and ZY designed the study, performed experiments and analyzed the data. XC was a major contributor in writing the manuscript. MJ and SY analyzed the data and revised the article. XS and RH designed the study and revised the article. All authors participated in the design of the experiments. All authors read and approved the final manuscript.

\section{Ethics approval and consent to participate}

This study was approved by the Ethics Committee of An Zhen Hospital of Capital Medical University (Beijing, China).

\section{Patient consent for publication}

Not applicable.

\section{Competing interests}

The authors declare that they have no competing interests.

\section{References}

1. Takagi Y, Yasuda S, Takahashi J, Tsunoda R, Ogata Y, Seki A, Sumiyoshi T, Matsui M, Goto T, Tanabe Y, et al: Clinical implications of provocation tests for coronary artery spasm: Safety, arrhythmic complications and prognostic impact: Multicentre registry study of the Japanese coronary spasm association. Eur Heart J 34: 258-267, 2013.

2. Yasue H, Nakagawa H, Itoh T, Harada E and Mizuno Y: Coronary artery spasm-clinical features, diagnosis, pathogenesis, and treatment. J Cardiol 51: 2-17, 2008.

3. Lanza GA, Careri G and Crea F: Mechanisms of coronary artery spasm. Circulation 124: 1774-1782, 2011.

4. Satoh S, Omura S, Inoue H, Mori T, Takenaka K, Numaguchi K, Mori E, Aso A, Nakamura T and Hiyamuta K: Clinical impact of coronary artery spasm in patients with no significant coronary stenosis in acute coronary syndromes. J Cardiol 61: 404-409, 2013.

5. FurchgottRF and Zawadzki JV: The obligatory role of endothelial cells in the relaxation of arterial smooth muscle by acetylcholine. Nature 288: 373-376, 1980.

6. Yamada E: The fine structure of the gall bladder epithelium of the mouse. J Biophys Biochem Cytol 1: 445-458, 1955.

7. Osugi T, Saitoh S, Matumoto K, Muto M, Aikawa K, Ohkawara H, Sugimoto K, Kamioka M, Ishibashi T and Maruyama Y: Preventive effect of chronic endothelin type A receptor antagonist on coronary microvascular spasm induced by repeated epicardial coronary artery endothelial denudation in pigs. J Atheroscler Thromb 17: 54-63, 2010.

8. Shiroto T, Romero N, Sugiyama T, Sartoretto JL, Kalwa H, Yan Z, Shimokawa H and Michel T: Caveolin-1 is a critical determinant of autophagy, metabolic switching, and oxidative stress in vascular endothelium. PLoS One 9: e87871, 2014.

9. Okamoto T, Schlegel A, Scherer PE and Lisanti MP: Caveolins, a family of scaffolding proteins for organizing 'preassembled signaling complexes' at the plasma membrane. J Biol Chem 273: 5419-5422, 1998

10. Grayson TH, Chadha PS, Bertrand PP, Chen H, Morris MJ, Senadheera S, Murphy TV and Sandow SL: Increased caveolae density and caveolin-1 expression accompany impaired $\mathrm{NO}$-mediated vasorelaxation in diet-induced obesity. Histochem Cell Biol 139: 309-321, 2013.

11. Bucci M, Gratton JP, Rudic RD, Acevedo L, Roviezzo F, Cirino G and Sessa WC: In vivo delivery of the caveolin-1 scaffolding domain inhibits nitric oxide synthesis and reduces inflammation. Nat Med 6: 1362-1367, 2000.

12. Weller R, Dykhuizen R, Leifert C and Ormerod A: Nitric oxide release accounts for the reduced incidence of cutaneous infections in psoriasis. J Am Acad Dermatol 36: 281-282, 1997.

13. Saitoh S, Takeishi Y and Maruyama Y: Mechanistic insights of coronary vasospasm and new therapeutic approaches. Fukushima J Med Sci 61: 1-12, 2015.

14. Gratton JP, Bernatchez P and Sessa WC: Caveolae and caveolins in the cardiovascular system. Circ Res 94: 1408-1417, 2004.

15. Maniatis NA, Shinin V, Schraufnagel DE, Okada S, Vogel SM, Malik AB and Minshall RD: Increased pulmonary vascular resistance and defective pulmonary artery filling in caveolin-1-/-mice. Am J Physiol Lung Cell Mol Physiol 294: L865-L873, 2008.

16. Ohi Y, Takai N, Muraki K, Watanabe M and Imaizumi Y: $\mathrm{Ca} 2+$-images of smooth muscle cells and endothelial cells in one confocal plane in femoral artery segments of the rat. Jpn J Pharmaclo 86: 106-113, 2001. 
17. Furchgott RF: The 1996 albert lasker medical research awards. The discovery of endothelium-derived relaxing factor and its importance in the identification of nitric oxide. JAMA 276 : $1186-1188,1996$

18. Smiljić S, Nestorović V and Savić S: Modulatory role of nitric oxide in cardiac performance. Med Pregl 67: 345-352, 2014.

19. Nilius B: Permeation properties of a non-selective cation channel in human vascular endothelial cells. Pflugers Arch 416: 609-611, 1990.

20. Newby AC and Henderson AH: Stimulus-secretion coupling in vascular endothelial cells. Annu Rev Physiol 52: 661-674, 1990.

21. Furchgott RF: Role of endothelium in responses of vascular smooth muscle. Circ Res 53: 557-573, 1983.

22. Shan LM and Wang H: Pharmacological characteristics of the endothelial target for acetylcholine induced vascular relaxation. Life Sci 70: 1285-1298, 2002.

23. Egashira K, Suzuki S, Hirooka Y, Kai H, Sugimachi M, Imaizumi $\mathrm{T}$ and Takeshita A: Impaired endothelium-dependent vasodilation of large epicardial and resistance coronary arteries in patients with essential hypertension. Different responses to acetylcholine and substance P. Hypertension 25 201-206, 1995.
24. Haga T: Molecular properties of muscarinic acetylcholine receptors. Proc Jpn Acad Ser B Phys Biol Sci 89: 226-256, 2013.

25. Hung MJ: Current advances in the understanding of coronary vasospasm. World J Cardiol 2: 34-42, 2010.

26. Drab M, Verkade P, Elger M, Kasper M, Lohn M, Lauterbach B, Menne J, Lindschau C, Mende F, Luft FC, et al: Loss of caveolae, vascular dysfunction, and pulmonary defects in caveolin-1 gene-disrupted mice. Science 293: 2449-2452, 2001

This work is licensed under a Creative Commons Attribution-NonCommercial-NoDerivatives 4.0 International (CC BY-NC-ND 4.0) License. 